\title{
Organic etiology of delusional parasitosis in the Hispanic population: a case report
}

ELENA EstradA ${ }^{1,2}$

Dharam Persaud-Sharma ${ }^{2}$

RAul GustaVo CORREDOR ${ }^{2}$

1 Citrus Psychiatry Health Network, Hialeah, FL, USA.

2 Florida International University, Herbert Wertheim College of Medicine, Miami, FL, USA.

Received: 11/08/2018 - Accepted: 02/15/2019

DOl: 10.1590/0101-60830000000193

Estrada E et al. / Arch Clin Psychiatry. 2019;46(2):51

\section{Dear Editor,}

Psychiatric conditions are prevalent amongst the geriatric population and are commonly managed by combined pharmacologic and behavioral therapy. In many cases, the symptoms of psychiatric conditions like delusions and hallucinations include set of diverse differential diagnoses, both organic and inorganic in nature. Thus, to properly identify the most likely etiology, an extensive medical workup must be performed to rule-out organic deficiencies, substance use disorder/iatrogenic toxicity, amongst other disorders leading to progressive neurocognitive decline. One must also consider a set of ethno-centric differential diagnoses. Here, we report a case of a patient with symptoms of delusional parasitosis refractory to conventional anti-psychotic pharmacotherapy, who experienced near total relief of symptoms after being treated for a cobalamin deficiency.

A 60-year old Hispanic woman with a past medical history of irritable bowel syndrome (IBS), hypothyroidism, migraines, and a 22-year psychiatric history of depression and anxiety who was initially admitted for depression and suicidal ideation because of unremitting delusional parasitosis. Prior pharmacotherapy with upward titrations of mirtazapine and fluphenazine, followed-by risperidone and venlafaxine, were all unsuccessful at reducing her symptoms of delusional parasitosis. Initial intake labs revealed cobalamin (B-12) deficiency without macrocytic anemia. Four days after intramuscular (IM) cobalamin injections, the patient's sensation of formication nearly completely resolved. Symptoms of delusional parasitosis were almost completely resolved at one-month follow-up.

Delusional parasitosis (DP) is a rare disorder where patients exhibit the fixed false belief of being infested with mites, parasites, or bugs despite the lack of empirical evidence supporting such symptoms with an estimated prevalence of 83.2 cases per million population per year and can negatively impact a patient's ability to function $^{1}$ and is classified as delusional disorder, somatic type in the DSM-52. It has been reported that patients with DP report bugs that are crawling in or on their skin, and will often apply pesticide to their wounds, use razor blades to try to remove insects and may attempt suicide ${ }^{3}$. Several of these features were observed with the patient, as she attempted to use scissors to try to cut the parasites from her skin and used Clorox bleach to clean her body of pests. In attempting to diagnose DP, organic causes such as dementia, head trauma, infarction, hyperthyroidism, anemia, lymphoma, tuberculosis, HIV, and vitamin B-12 deficiency should first be ruled out $^{1}$. In this case, Ms. A had undiagnosed vitamin B-12 deficiency which lead to the failure of her prior antidepressant and antipsychotic trials to relieve her DP.
The Institute of Medicine (IOM) recommends that people over age 50 consume vitamin B-12 from fortified food or supplement given the prevalence of vitamin B-12 deficiency in this population*4. Several studies have shown that in elderly Hispanics, the effects of vitamin B-12 deficiency are even more pronounced ${ }^{5-7}$. Elderly Hispanics aged 60-93 of Caribbean origin have been found to have lower vitamin B-12 levels when compared to Non-Hispanic whites ${ }^{5}$. When compared with Asians and African Americans, elderly Latin Americans have lower vitamin B-12 levels ${ }^{6}$. Possible explanations include increasing gastric dysfunction in age and higher gastrin level, or less vitamin B-12 intake in Hispanic populations $s^{5,7}$.

This case exemplifies that delusional parasitosis can result from undiagnosed vitamin B-12 deficiency and should be ruled-out prior to starting antipsychotic therapy; especially in patient's formication delusions. It also underlines the importance of ethno-specific differential diagnoses when providing psychiatric evaluation in the Hispanic population, as organic etiologies may be primarily responsible for many psychological manifestations of disease.

\section{References}

1. Suh KN, Keystone JS. Delusional parasitosis, in tropical infectious diseases: principles pathogens and practice. New York: Saunders; 2011. p. 1066-71.

2. American Psychiatric Association. Diagnostic and statistical manual of mental disorders: DSM-5. 5th ed. Washington, DC: American Psychiatric Association; 2013.

3. Aw DC, Thong JY, Chan HL. Delusional parasitosis: case series of 8 patients and review of the literature. Ann Acad Med Singapore. 2004;33(1):89-94.

4. Dietary Reference Intakes for Thiamin, Riboflavin, Niacin, Vitamin B6, Folate, Vitamin B12, Pantothenic Acid, Biotin, and Choline, in Institute of Medicine (US) Standing Committee on the Scientific Evaluation of Dietary Reference Intakes and its Panel on Folate, Other B Vitamins, and Choline. Washington, DC: National Academies Press; 1998.

5. Kwan LL, Bermudez OI, Tucker KL. Low vitamin B-12 intake and status are more prevalent in Hispanic older adults of Caribbean origin than in neighborhood-matched non-Hispanic whites. J Nutr. 2002;132(7):2059-64.

6. Carmel R, Green R, Jacobsen DW, Rasmussen K, Florea M, Azen C. Serum cobalamin, homocysteine, and methylmalonic acid concentrations in a multiethnic elderly population: ethnic and sex differences in cobalamin and metabolite abnormalities. Am J Clin Nutr. 1999;70(5):904-10.

7. Campbell AK, Miller JW, Green R, Haan MN, Allen LH. Plasma vitamin B-12 concentrations in an elderly Latino population are predicted by serum gastrin concentrations and crystalline vitamin B-12 intake. J Nutr. 2003;133(9):2770-6. 